\title{
Lithium abundances in the old open cluster NGC 3960 from VLT/FLAMES observations ${ }^{\star, \star \star}$
}

\author{
L. Prisinzano ${ }^{1}$ and S. Randich ${ }^{2}$ \\ 1 INAF - Osservatorio Astronomico di Palermo, Piazza del Parlamento 1, 90134 Palermo, Italy \\ e-mail: loredana@astropa.inaf.it \\ 2 INAF - Osservatorio Astronomico di Arcetri, Largo E. Fermi 5, 50125 Firenze, Italy \\ e-mail: randich@arcetri.astro.it
}

Received 24 July 2007 / Accepted 7 September 2007

ABSTRACT

\begin{abstract}
Context. Old open clusters are very useful targets to investigate mechanisms responsible for lithium (Li) depletion during the main sequence. Comparison of the $\mathrm{Li}$ abundances in clusters of different age allows us to understand the efficiency of the Li destruction process.

Aims. To determine the membership and Li abundance in a sample of candidate members of the open cluster NGC 3960 (age 1 Gyr), aiming to fill the gap between 0.6 and $2 \mathrm{Gyr}$ in the empirical description of the behavior of the average $\mathrm{Li}$ abundance as a function of the stellar age.

Methods. We use VLT/FLAMES Giraffe spectra to determine the radial velocities and thus the membership of a sample of 113 photometrically selected candidate cluster members. From the analysis of the Li line we derive Li abundances for both cluster members and non-members.

Results. 39 stars have radial velocities consistent with membership, with an expected fraction of contaminating field stars of about $20 \%$. Li is detected in 29 of the radial velocity members; we consider these stars as cluster members, while we make the reasonable assumption that the remaining 10 radial velocity members without $\mathrm{Li}$ are among the contaminating stars. Li abundances of the stars hotter than about $6000 \mathrm{~K}$ are similar to those of stars in the Hyades, while they are slightly smaller for cooler stars. This confirms that NGC 3960 is older than the Hyades.

Conclusions. The average $\mathrm{Li}$ abundance of stars cooler than about $6000 \mathrm{~K}$ indicates that the Li Pop. I plateau might start at $\sim 1 \mathrm{Gyr}$, rather than $2 \mathrm{Gyr}$, which is the upper limit previously derived in the literature. We also find that the fraction of field stars with high $\mathrm{Li}$ abundance $(\gtrsim 1.5)$ is about one third of the whole sample, which is in agreement with previous estimates. The fraction of contaminating field stars is consistent with that previously derived by us from photometry.
\end{abstract}

Key words. stars: abundances - stars: evolution - Galaxy: open clusters and associations: individual: NGC 3960

\section{Introduction}

Open cluster (OCs) are commonly recognized as one of the best tools to investigate the formation and evolution of the Galactic disk, as well as the evolution of stars and their properties.

We have carried out a VLT/FLAMES project aimed at deriving homogeneous information for a sample of 11 old OCs (ages greater than about 0.9 Gyr) with the aim of a) deriving their chemical composition from UVES spectra of evolved stars, and b) determining their radial velocities and thus membership, together with lithium (Li) abundances from Giraffe spectra of turn-off (TO) and main sequence (MS) cluster candidates (Randich et al. 2005; Pallavicini et al. 2006). In this paper we focus on Giraffe observations of NGC 3960, the youngest OC in our sample.

In the last few years, this cluster has been the object of different photometric and spectroscopic studies, which have allowed a more accurate determination of its parameters. Bragaglia et al. (2006) derived an age between 0.6 and $0.9 \mathrm{Gyr}$, a distance modulus $(m-M)_{0}=11.6 \pm 0.1$, a reddening $E(B-V)=0.29 \pm 0.02$,

* Based on observations collected at ESO-VLT, Paranal Observatory, Chile, Programme numbers 73.D-0520(A)

$\star \star$ Table 4 is only available in electronic form at http://www. aanda.org with differential reddening $\Delta E(B-V)=0.05$. A slightly older age (in the range 0.9-1.4 Gyr) was derived by Prisinzano et al. (2004) who also estimated the cluster mass function from the luminosity function in the $V$ and $J$ bands, finding a slope $\alpha=$ $2.95 \pm 0.53$ for masses above $1 M_{\odot}$. Note that the contamination from field stars was taken into account using a control field and performing a statistical subtraction. A spectroscopic $[\mathrm{Fe} / \mathrm{H}]=-0.12 \pm 0.04$ was derived by Bragaglia et al. (2006), while Sestito et al. (2006) report a slightly higher value $[\mathrm{Fe} / \mathrm{H}]=0.02 \pm 0.04$ : in both cases the metallicity is consistent with the solar value.

Given its age (we assume here $0.9 \mathrm{Gyr}$ ), NGC 3960 provides a good sample to investigate the evolution of lithium abundance during the MS and, in particular, to fill the gap in age coverage between the Hyades (0.6 Gyr) and the $~ 1.5-2.0$ Gyr clusters for which Li data are available (NGC 752, IC 4651).

Sestito \& Randich (2005) investigated the timescales for $\mathrm{Li}$ depletion during the MS for F and G-type stars, by means of a homogeneous re-analysis of $\mathrm{Li}$ data for several open clusters. They suggested that $\mathrm{Li}$ depletion is not a continuous process, but is instead characterized by different timescales in different age intervals. In particular, they showed that Li depletion slows down after the Hyades age for stars in the temperature range $\sim 6050-6350 \mathrm{~K}$, while it stops for cooler stars. Namely, for stars 
cooler than $\sim 6050 \mathrm{~K}$, a plateau in Li abundance is seen for ages older than $\sim 2$ Gyr; this age, however, represents an upper limit to the actual age when Li depletion is no longer efficient. A more precise estimate of this age, which would provide a useful constraint to models including extra-mixing during the MS, requires Li data for clusters with ages between 0.6-2 Gyr. We also mention in passing that since the study of Sestito \& Randich (2005) has been used to infer the age of stars hosting extra-solar planets (e.g. Sozzetti et al. 2007), a finer sampling of the 0.6-2 Gyr interval would allow these stars to be age dated with higher accuracy.

Besides allowing measurements of $\mathrm{Li}$ abundances, our Giraffe spectra have been used to determine radial velocities and cluster membership for the observed candidates. This in turn will allow us to perform a revised analysis of contamination from field stars and cluster mass function.

Our paper is structured as follows: in Sect. 2 we describe the criterion adopted to select the spectroscopic targets and observations, and in Sect. 3 we report the analysis of the spectra, including the procedure used to derive the radial velocity $(R V)$ and the equivalent width $(E W)$ of the Li line. In Sect. 4 we describe how we derive the effective temperatures and the Li abundances of our targets. In Sect. 5, we discuss the Li abundances for both the cluster members and the field stars, by comparing our results with those of stars of similar spectral type taken from literature. We also compare the fraction of contaminating field stars in the spectroscopic sample with that previously obtained statistically from photometric data. Our conclusions are presented in Sect. 7.

\section{Target selection and observations}

The targets observed with VLT/FLAMES were retrieved from the photometric catalog of Prisinzano et al. (2004), selecting objects in the cluster region, i.e., within $7^{\prime}$ from the cluster centroid. In total, we observed 113 candidate members on the MS with $16 \leq V \leq 18$, corresponding to spectral-types from $\mathrm{F}$ to early K: stars with $V$ larger than 18 are too cool and faint to reach a $S / N$ ratio larger than 40, which is required to accurately measure the $R V \mathrm{~s}$ and $E W \mathrm{~s}$; stars brighter than 16 are hotter than early F stars and therefore are not good targets to investigate $\mathrm{Li}$ depletion in old MS stars.

In Fig. 1 we show the $V$ vs. $V-I$ color-magnitude diagram for the region within $7^{\prime}$ from the centroid of NGC 3960 (dots) where photometry is corrected for differential reddening.

The observations were carried out in Service Mode during February, March and April 2004; the log of the observations is provided in Table 1 . The cluster was covered by one configuration centered at $\operatorname{RA}(2000)=11^{\mathrm{h}} 50^{\mathrm{m}} 31^{\mathrm{s}} .400$ $\operatorname{Dec}(2000)=-55^{\circ} 41^{\prime} 12^{\prime \prime} .80$. Giraffe was used in conjunction with the 316 lines $/ \mathrm{mm}$ grating and order sorting filters 14 (HR14) and 15 (HR15), yielding nominal resolving powers $R=28800$ and 19300, respectively. Spectral coverages are from 630.8 to 670.1 for HR14 and from 660.7 to $696.5 \mathrm{~nm}$ for HR15; they include $\mathrm{H} \alpha$, the $\mathrm{Li}$ I $670.8 \mathrm{~nm}$ line and several features to be used for $R V$ measurements. For each set-up, four $45 \mathrm{~min}$ exposures were obtained.

Data reduction was carried out using the GIRAFFE BLDRS pipeline $^{1}$, following the standard procedure and steps (Blecha \& Simond 2004). Sky subtraction for the spectrum of each set-up and each exposure was performed separately, namely by subtracting the median, computed as in Jeffries \& Oliveira (2005), of the 16 sky fiber spectra observed in the same exposure. The four

\footnotetext{
1 Version 1.0 - http://girbldrs. sourceforge.net/
}

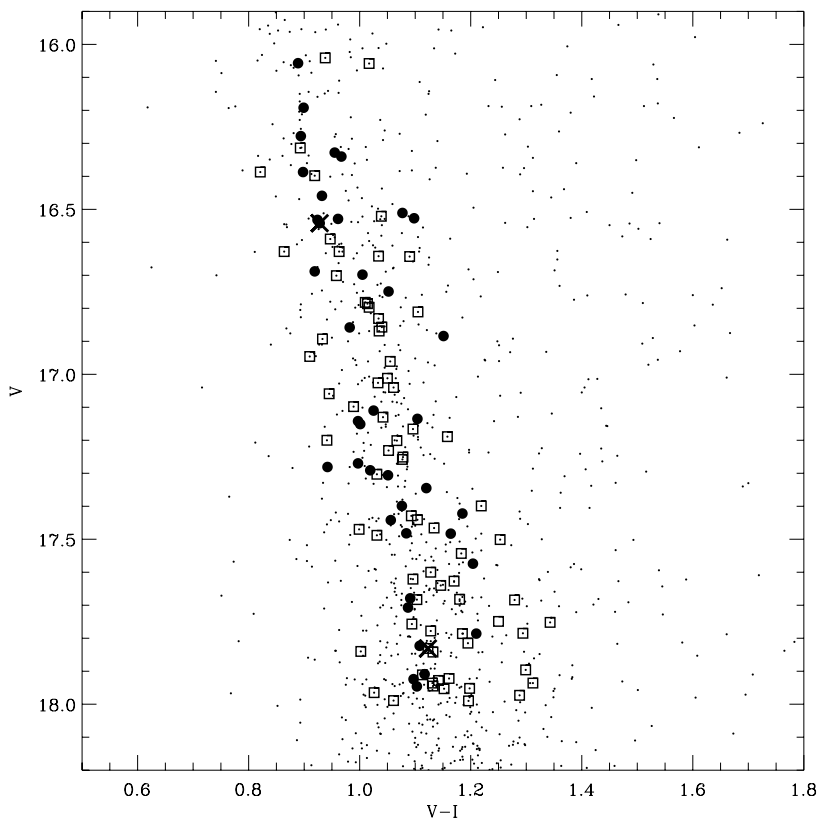

Fig. 1. Color-magnitude diagram for the region within $7^{\prime}$ from the centroid of NGC 3960 (dots). Photometry is from Prisinzano et al. (2004) and it is corrected for differential reddening. Targets observed with VLT/FLAMES are indicated with large symbols: filled circles are those with $R V$ consistent with that of the cluster and empty squares are non-members having the $R V$ different from the cluster members (see Sect. 3.1.1). The two stars indicated with the $\mathrm{X}$ symbols are candidate spectroscopic binaries (SB2).

Table 1. Log-book of Giraffe/FLAMES observations. Columns 1-4 give the observation date, original name associated with each observing block, exposure time, and grating used for each exposure, respectively.

\begin{tabular}{cccc}
\hline \hline $\begin{array}{c}\text { Obs. } \\
\text { Date }\end{array}$ & $\begin{array}{c}\text { Exp. } \\
\text { Name }\end{array}$ & $\begin{array}{c}\text { Exp. Time } \\
\text { (s) }\end{array}$ & Grating \\
\hline $04 / 03 / 2004$ & M1-Li-a & 2700 & HR15 \\
$04 / 03 / 2004$ & M2-Li-a & 2700 & HR15 \\
$04 / 03 / 2004$ & M2-Li-c & 2700 & HR15 \\
$04 / 20 / 2004$ & M1-ha & 2700 & HR14 \\
$05 / 02 / 2004$ & M1-Li-b & 2700 & HR15 \\
$05 / 02 / 2004$ & M2-ha-a & 2700 & HR14 \\
$05 / 02 / 2004$ & M2-ha-b & 2700 & HR14 \\
$05 / 20 / 2004$ & M2-ha-c & 2700 & HR14 \\
\hline
\end{tabular}

sky-subtracted spectra of each target obtained with the HR15 set-up were then co-added, after applying the Doppler correction due to the different date of observations. Examples of final co-added sky-subtracted spectra around the Li line are shown in Fig. 2. $S / N$ ratios are $\sim 80$ for the stars OC21-M342 and OC21M42 and $~ 55$ for OC21-M205 and OC21-M359. Final $S / N$ ratios range between 30 and 80 , as estimated from the faintest and the brightest stars in our sample.

\section{Data analysis}

\subsection{Radial velocities}

$R V \mathrm{~s}$ in the heliocentric system of the 8 sets of spectra were computed using the function giCrossC of the GIRAFFE girBLDR pipeline. We computed the cross-correlation function between all the observed spectra and the synthetic template spectrum girKO corresponding to a $\mathrm{K} 0 \mathrm{~V}$ star. We used a delta $R V$ limit to the cross-correlation window of $500 \mathrm{~km} \mathrm{~s}^{-1}$ in the wavelength 

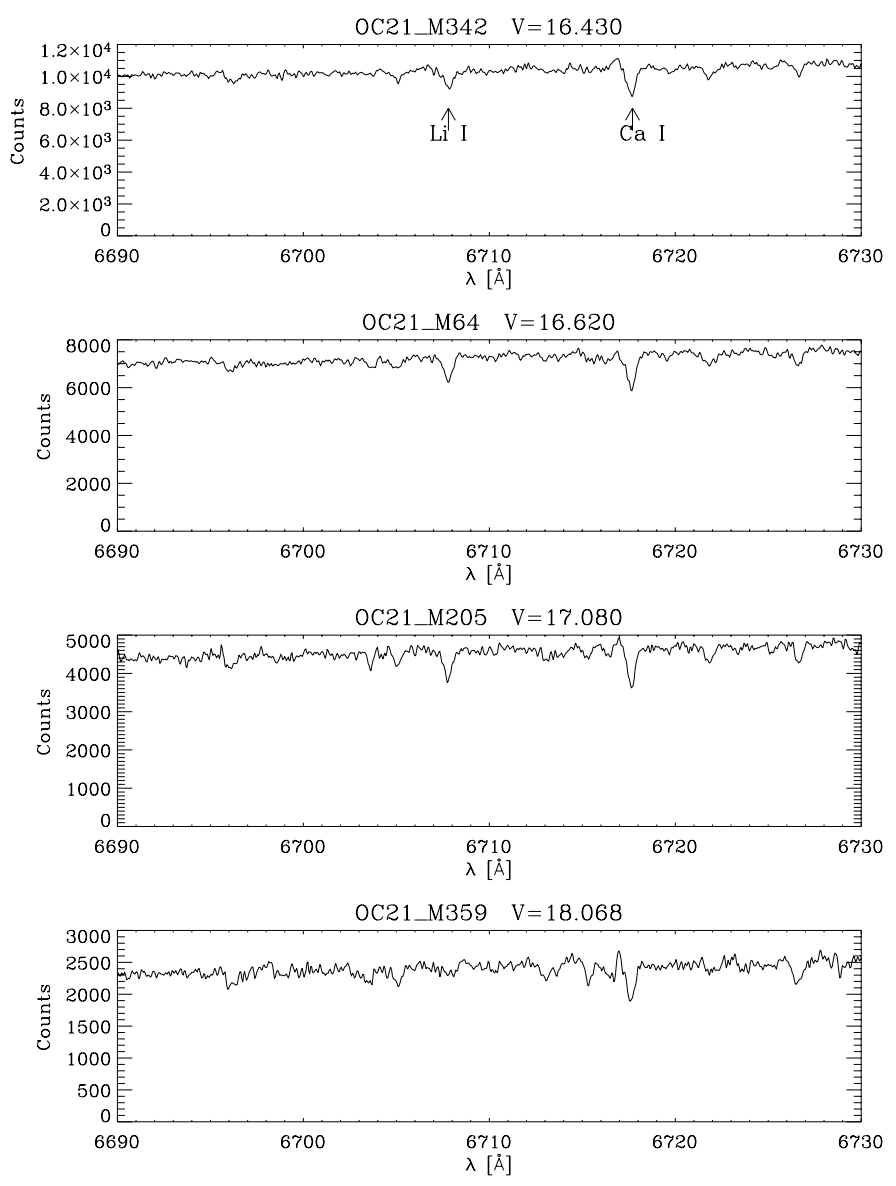

Fig. 2. Examples of co-added sky-subtracted spectra in the spectral region that includes the Li line. The GIRAFFE ID given in Table 4 and the $V$ magnitude of the stars are indicated.

ranges $[6310-6680] \AA$, for the spectra around the Li line, and [6610-6810] $\AA$, for the spectra around the $\mathrm{H} \alpha$ line.

Final $R V \mathrm{~s}$ for the stars of our sample were computed as the mean value and the standard deviation of the $R V \mathrm{~s}$ obtained with giCrossC from the 8 different sets of spectra.

The $R V$ s obtained using the function giCrossC as described before were compared with those obtained by using a different template spectrum, girG2, which corresponds to a spectrum of a solar-type star. The comparison does not evidence significant differences. In addition, in order to check the reliability of the automatic cross-correlation performed by the pipeline, we computed the $R V$ for 4 of the 8 sets of spectra using the $\operatorname{IRAF}^{2}$ task FXCOR (Tonry \& Davis 1979); as template we used the spectrum of a relatively bright star $(V=16.5$, spectral type F8V) from our sample, which is considered a reliable cluster member, based on its $R V$ as measured with gicrossC. The relative $R V \mathrm{~s}$ computed with FXCOR were converted into the heliocentric system using the heliocentric $R V$ of the template star computed with giCrossC. The median of the differences between the four sets of $R V \mathrm{~s}$ obtained with giCrossC $R V \mathrm{~s}$ minus FXCOR $R V \mathrm{~s}$ are $0.13,-0.34,0.12$ and $-1.05 \mathrm{Km} \mathrm{s}^{-1}$, while the standard deviations are $2.13,2.16,1.81$ and $2.10 \mathrm{Km} \mathrm{s}^{-1}$. Such values indicate an excellent agreement between the two methods. The difference between the mean $R V \mathrm{~s}$ obtained with the two methods

${ }^{2}$ IRAF is distributed by the National Optical Astronomical Observatories, which are operated by the Association of Universities for Research in Astronomy, under contract with the National Science Foundation. shows that only 8 stars (OC21-M80, OC21-M273, OC21-M581, OC21-M324, OC21-M282, OC21-M185, OC21-M177, OC21M41) have a difference in the mean $R V$ obtained with the two methods larger than $5 \mathrm{~km} \mathrm{~s}^{-1}$ and only 2 (OC21-M80 and OC21$\mathrm{M} 282)$ have a difference larger than $10 \mathrm{~km} \mathrm{~s}^{-1}$. These objects do not show the $\mathrm{Li}$ line and 7 of them are not cluster members since they have a $R V$ not consistent with that of the cluster (see next section). The only star classified as a cluster member (OC21M273) based on its $R V$ does not show the Li line and therefore it is considered a contaminating field star.

For 2 stars (OC21-M286, OC21-M136) the peak of the cross-correlation function performed with FXCOR is not symmetric but shows a double peak, which indicates that these objects are double-lined spectroscopic binaries (SB2). An inspection of the spectrum of these objects confirms the double spectral features, typical of SB2 stars.

\subsubsection{Cluster membership}

The density distribution of the final $R V \mathrm{~s}$ is shown in the histogram of Fig. 3, where a significant peak around $R V=$ $-20 \mathrm{~km} \mathrm{~s}^{-1}$ indicates the presence of the cluster with respect to the $R V$ distribution of the field stars, which shows a secondary peak at about $-2 \mathrm{~km} \mathrm{~s}^{-1}$.

To derive the cluster membership based on the $R V$ we fitted the final $R V$ distribution with a double Gaussian using the "maximum likelihood fitting", as in Prisinzano et al. (2007). We find that the cluster Gaussian is centered on $-20.0 \pm 0.7 \mathrm{~km} \mathrm{~s}^{-1}$ with a standard deviation $\sigma=2.3 \pm 0.6 \mathrm{~km} \mathrm{~s}^{-1}$, while the much broader field star $R V$ distribution shows a peak at $-2.1 \pm 2.6 \mathrm{~km} \mathrm{~s}^{-1}$, with a standard deviation of $\sigma=22.9 \pm 1.8 \mathrm{~km} \mathrm{~s}^{-1}$. The fitted curve is indicated by the solid line in Fig. 3. We note that the average cluster $R V$ is smaller than the mean $R V$ of NGC 3960 (equal to about $-12 \mathrm{~km} \mathrm{~s}^{-1}$ ) computed from 5 members studied in Friel \& Janes (1993), but in very good agreement with the value derived by Sestito et al. (2006) from UVES spectra $\left(-22.6 \pm 0.9 \mathrm{~km} \mathrm{~s}^{-1}\right)$.

The total number of possible cluster members within $\pm 3 \sigma$ of the cluster $R V$ distribution is 39 including 16 contaminating field stars, as computed from the Gaussian distribution of the field stars. We note that some of the classified non-members could be spectroscopic binaries of the cluster, which cannot be easily distinguished from true non-members. From an inspection of the single spectra, only two objects, indicated by $\mathrm{X}$ symbols in Fig. 1, have been recognized from the characteristic double-line spectrum typical of SB2 stars (see Sect. 3.1).

Note that the identification of the cluster members does not allow us to reduce the spread in the CMD since the differential reddening corrections are computed using the reddening map shown in Fig. 8 of Prisinzano et al. (2004), which gives the relative reddening values in subregions of $1^{\prime} .7 \times 1^{\prime} .7$, with respect to the subregion where the cluster centroid is located. They are therefore spatially-dependent corrections and are not calculated for each star individually. This statistical method also includes the contaminating field stars. Individual spectral type estimates from low resolution spectra are needed to derive the individual reddening and thus the accurate position of the members in the CMD.

\subsection{Li equivalent widths}

Li $E W s$ were measured on the co-added spectra normalized to their continuum. The normalization was performed using the region of the spectrum between 6693 and $6722 \AA$ that includes the 


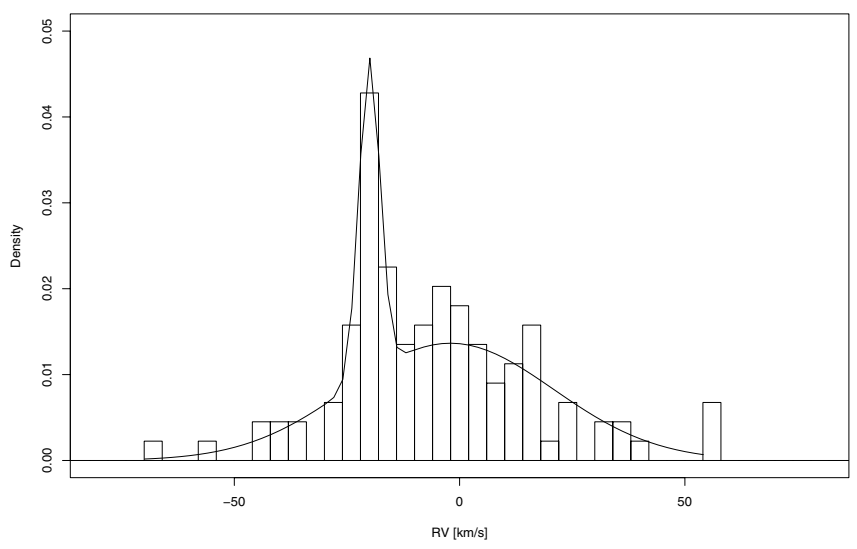

Fig. 3. Density distribution of the $R V$ of the whole sample, which includes 113 stars. The fitted double Gaussian (see text) is indicated by the solid line.

Li I $6707.8 \AA$ line; we used the IRAF task CONTINUUM with a second order Legendre function and a variable residual rejection limit chosen based on the visual inspection of the fitting result. The $E W$ s of the Li I $6707.8 \AA$ line were measured using the IRAF task SPLOT, assuming a Gaussian profile, which is a good approximation since the $E W \mathrm{~s}$ are all smaller than about $100 \mathrm{~m} \AA$ and therefore the line is not saturated.

The continuum normalization and the $E W$ measurements were repeated three times, on different dates; the continuum has been estimated by choosing each time a different residual rejection limit, that causes a change of the continuum level; different measures have been done after few months and therefore they are quite different. The mean of the three measurements was taken as the final estimation of the $E W$, and the maximum uncertainty was used as the error. We detected the Li line in 55 objects, 29 with $R V \mathrm{~s}$ that were consistent with membership and 26 with $R V \mathrm{~s}$ corresponding to non-members. Among the remaining 58 stars, we have 56 single objects and two SB2 binaries. For the 56 single stars, we estimated an upper limit of the $E W$ by measuring the $E W$ of the smallest line around $6707.8 \AA$. In some cases the spectra are of candidate $\mathrm{M}$ dwarf stars and show large molecular bands around the $\mathrm{Li}$ line. For these stars, we considered the whole depression of the spectrum, which includes the Li line, therefore the upper limits are very conservative.

We corrected measured $E W \mathrm{~s}$ for the contribution of the $\mathrm{Fe} \mathrm{I}$ line at $6707.44 \AA$ using the relation $E W(\mathrm{Fe})=20(B-V)_{0}-3 \mathrm{~m} \AA$, given in Soderblom et al. (1993a) for stars with solar metallicity, thus appropriate for our cluster (Sestito et al. 2006).

Figure 4a shows the Li EWs measured for all of the 55 objects where $\mathrm{Li}$ was detected as a function of the dereddened $(B-V)$ colors, corrected for differential reddening, as described in Prisinzano et al. (2004). Figure 4b shows the Li $E W$ s for the stars that are cluster members based on their $R V$, while Fig. $4 \mathrm{c}$ shows the Li $E W$ s for the non-members. Upper limits are also plotted in the three panels, as well as Li $E W$ s measured for the stars in the Hyades (Soderblom et al. 1990; Thorburn et al. 1993; Soderblom et al. 1995) .

Whereas our measurements are characterized by a larger spread, Fig. $4 \mathrm{~b}$ indicates that the Li $E W \mathrm{~s}$ of $R V$ members are on average smaller than those of the Hyades members of similar color. This is also true for most of the $R V$ non-members (Fig. 4c) with $(B-V)_{0}$ smaller than about 0.6 (F and G-type stars), while for redder colors, Li $E W \mathrm{~s}$ are more comparable with those of Hyades stars.
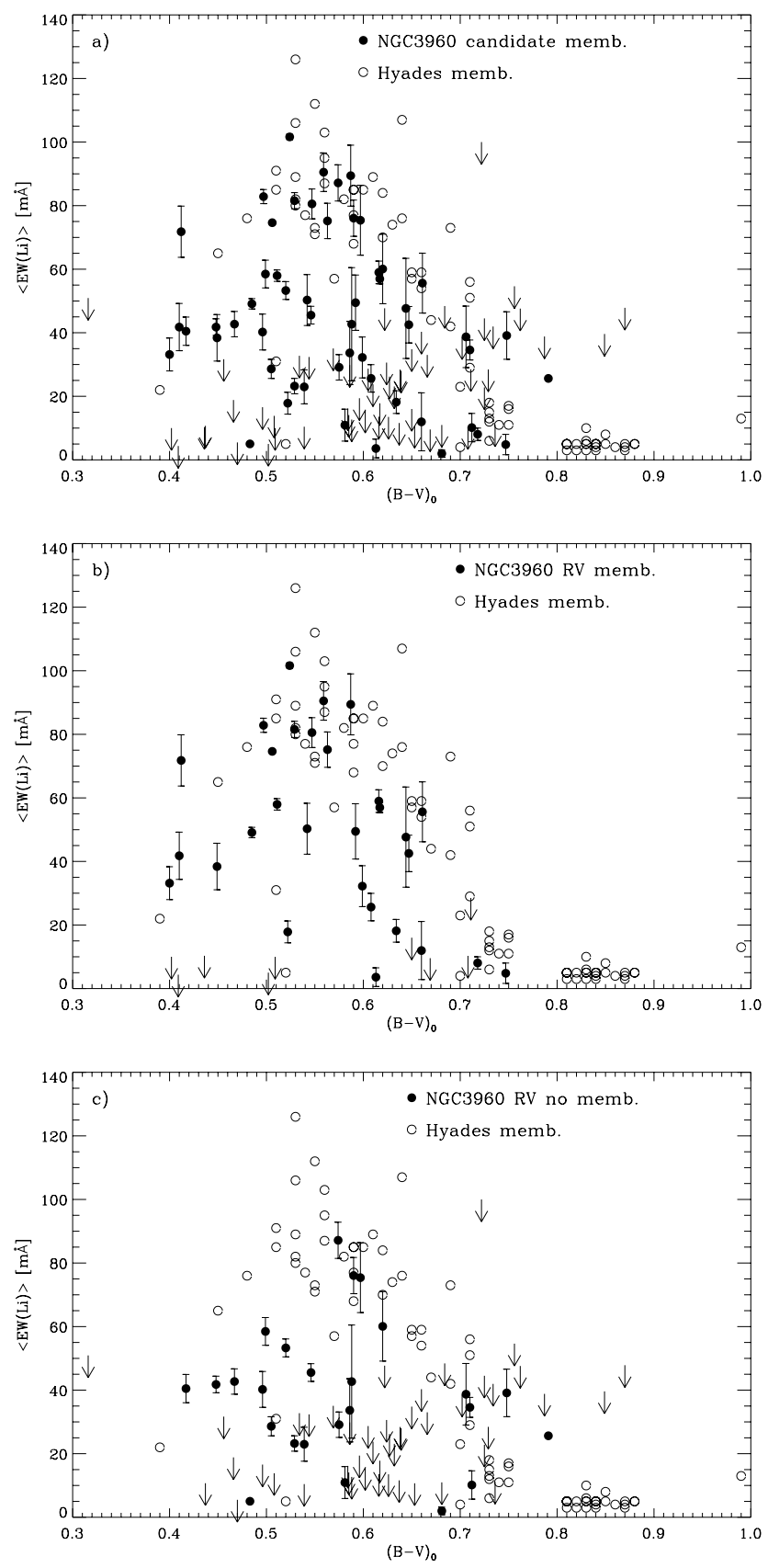

Fig. 4. a) Li $E W \mathrm{~s}$ and upper limits to $E W \mathrm{~s}$ for all our candidate members as a function of the dereddened $(B-V)$ colors, corrected for differential reddening, as described in Prisinzano et al. (2004). Panels b) and c) show the Li $E W \mathrm{~s}$ for the subsamples of the candidate members that are cluster members (panel b)) or contaminating field stars (panel c)), based on their $R V$. Our measurements are compared with the $E W \mathrm{~s}$ measured for the stars in the Hyades (Sestito \& Randich 2005).

\section{Li analysis}

\subsection{Effective temperatures}

As in Sestito \& Randich (2005), effective temperatures were computed from the dereddened $(B-V)$ colors, using the relation $T_{\text {eff }}=1800(B-V)_{0}^{2}-6103(B-V)_{0}+8899 \mathrm{~K}$, given by Soderblom et al. (1993b). Since photometric errors are smaller than $0.01 \mathrm{mag}$, errors in the effective temperatures are mainly due to the interstellar reddening correction. Indeed, as discussed in Prisinzano et al. (2004), Bragaglia et al. (2006), Bonatto \& Bica (2006), NGC 3960 is affected by a relatively strong 

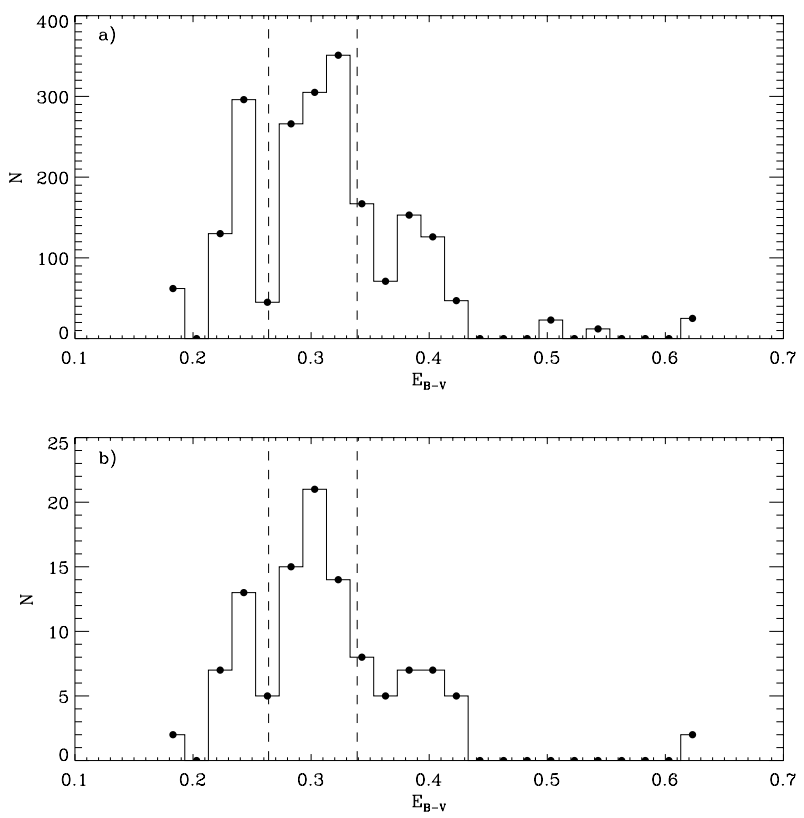

Fig. 5. Reddening distribution computed as described in Prisinzano et al. (2004), for all stars within 7 arcmin from the cluster center (panel a)) and for the stars observed with Giraffe (panel b)). The dashed lines indicate the 25th (0.26) and the 75th (0.34) percentiles of the two distributions (see text).

differential reddening. Figure 5 shows the reddening distribution computed as described in Prisinzano et al. (2004), for all stars within 7 arcmin from the cluster center (Fig. 5a) and for the stars observed with Giraffe (Fig. 5b). The dashed lines indicate the 25th (0.26) and the 75th (0.34) percentiles of the two distributions around the median value equal to 0.30 ; we assume the semi-difference of these two percentiles, equal to 0.04 , as the typical error of $E(B-V)$. This range is in agreement with $0.29 \pm 0.05$ found in Bragaglia et al. (2006) and with the range $[0.03,0.34]$ given by Bonatto \& Bica (2006).

Assuming that $\sigma_{(B-V)} \sim \sigma_{E(B-V)}$, i.e., the error in the $(B-V)$ colors corrected for differential reddening is of the order of the reddening error, we computed errors in the effective temperatures as the propagated uncertainties.

\subsection{Li abundances}

Li abundances were computed from the $E W$ s and the effective temperatures by interpolating the growth curves of Soderblom et al. (1993a). Since these curves are computed assuming the local thermodynamic equilibrium (LTE), we corrected the derived Li abundance, for non-local thermodynamic equilibrium (NLTE) using the Carlsson et al. (1994) code.

Errors in Li abundances were estimated by independently computing errors in Li abundance due to the effective temperature errors and $E W$ errors. Finally, we quadratically added these errors to estimate our uncertainties in $\mathrm{Li}$ abundances.

The computed $\mathrm{Li}$ abundances as a function of the temperatures for all the photometric candidates are shown in Fig. 6a; the $R V$ members and the non-members are shown separately in Figs. $6 \mathrm{~b}$ and c, respectively. Upper limits are also plotted. Final data are given in Table 4 where we list the following: identification number of Prisinzano et al. (2004), Giraffe identification name and spectrum number, celestial coordinates, $V$ and $B$ magnitudes, $V$ and $B$ magnitudes corrected for differential reddening, membership flag based on the $R V$ (0 means "non-member",
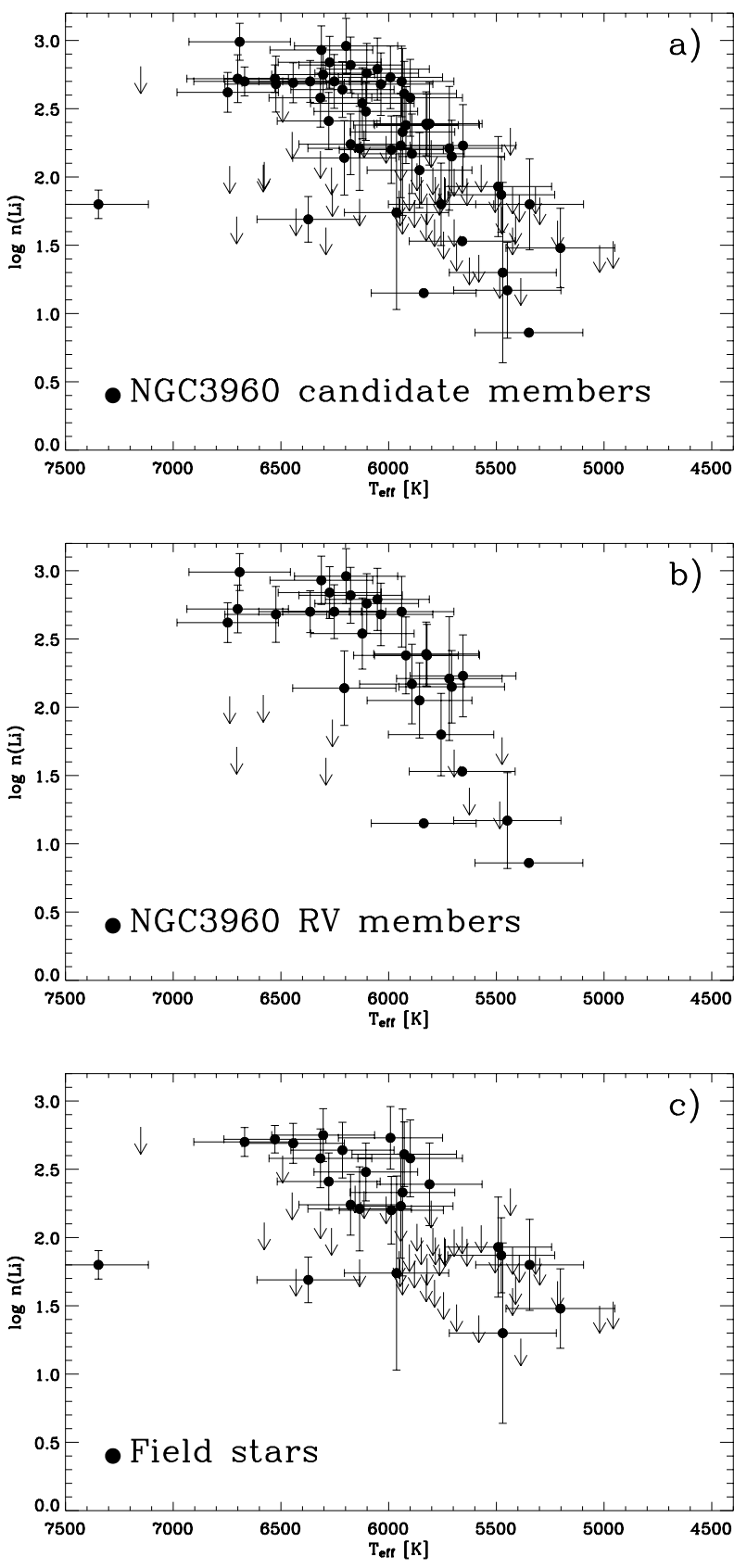

Fig. 6. Li abundances derived from the effective temperatures computed using the $(B-V)_{0}$ colors corrected for differential reddening, as described in Prisinzano et al. (2004). As in Fig. 4, the values are reported for the whole sample of photometric candidate members (panel a)) and for the subsamples of $R V$ members and non-members (panels $\mathbf{b}$ ) and $\mathbf{c}$ ), respectively). Upper limits for the stars without $\mathrm{Li}$ are also indicated.

1 means "member" and 2 means "binary"), effective temperatures computed from the $(B-V)_{0}$ colors, corrected for differential reddening, $E W$ of the Li line, and finally LTE and NLTE Li abundances. Note that in some cases, the NLTE Li abundances were not computed because the LTE Li abundances were outside the range of allowed values for the Carlsson et al. (1994) correction. Note also that for the 2 binaries, the $E W$ of the $\mathrm{Li}$ line were not measured due to the complexity of the spectrum of these objects.

For comparison, Fig. 7 shows the same plots of Fig. 6, but with the Li abundances derived from the effective temperatures 

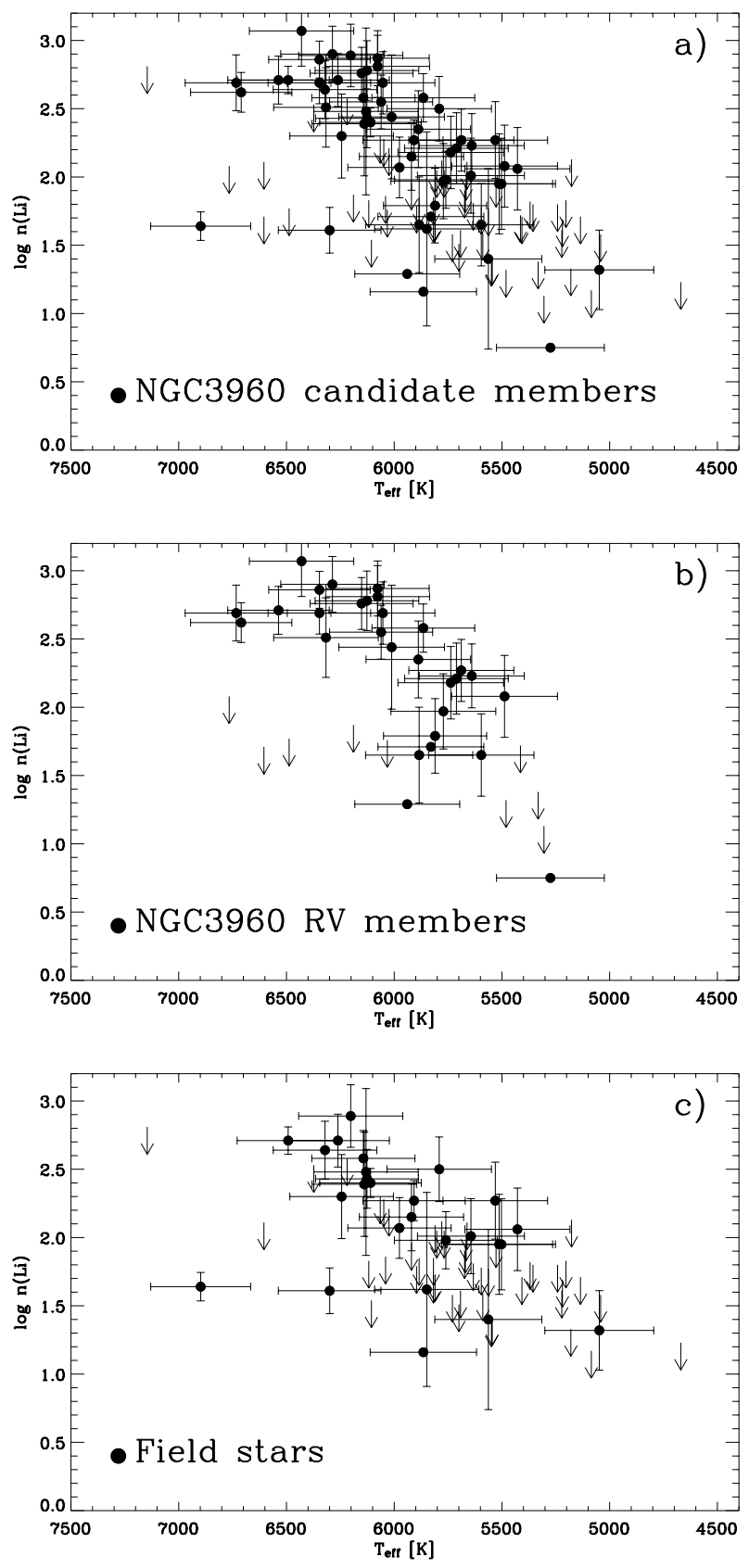

Fig. 7. Li abundances derived from the effective temperatures computed using the $(B-V)_{0}$ colors not corrected for differential reddening. The reported subsamples are the same described in Fig. 6.

computed using the $(B-V)_{0}$ colors not corrected for differential reddening.

We note that $\mathrm{Li}$ abundances in Fig. $7 \mathrm{~b}$ are slightly more spread out than those in Fig. 6b. This suggests that temperatures obtained from colors corrected for differential reddening are, on average, more accurate than those from uncorrected colors; hence, we adopt them for the following analysis. However, the adopted reddening corrections are derived statistically from photometry assuming the distance of the stars of NGC 3960, therefore, the reddening correction may not be appropriate for the field stars, especially if they are foreground field stars. Nevertheless, we find that the spread in Fig. 6c is smaller than that shown in Fig. 7c and similar to that found in Pasquini et al. (1994) for field stars of similar temperatures.

\section{Discussion}

\subsection{Li abundances}

Figure 6a evidences a large spread in the Li abundance distribution: it includes both the coeval $R V$ members of NGC 3960 and the inhomogeneous sample of $R V$ non-members. This is especially true for F-types and later spectral types, where the amount of the Li depletion significantly depends on stellar age. In the following sections we focus on the $\mathrm{Li}$ distribution for cluster members and likely field stars.

\subsubsection{Cluster members}

Figure 6 b shows the $\mathrm{Li}$ abundance distribution for the 29 stars with detected $\mathrm{Li}$ line and $R V$ s consistent with membership. As already mentioned in Sect. 3.1, we estimate that a total of 39 stars are $R V$ members, over a total of 113 objects studied in this work. Of the 39 objects, 16 are expected to be contaminating field stars, with $R V$ s consistent with that of the cluster.

Based on Li only, we cannot definitively rule out the possibility that the 10 stars with $R V$ s consistent with membership and without $\mathrm{Li}$ are cluster members ${ }^{3}$. On the one hand, for some of them we have inferred upper limit values comparable or even higher than measured Li $E W$ s of stars with detected Li. On the other hand, considering these 10 objects as cluster members would imply a large spread in the Li abundances for warmer stars, which, again, we cannot completely exclude. We note however that if these 10 stars, or most of them, were indeed cluster members, most of the contaminating field stars would have high $\mathrm{Li}$, which is rather unlikely. On the contrary, we make the more reasonable hypothesis that the $29 R V$ members with detected $\mathrm{Li}$ are cluster members, while the 10 remaining objects labeled as $R V$ members, but without a detected Li line, are among the 16 contaminating stars. This assumption would imply that we have $\sim 60 \%$ of non-members $(10 / 16)$ without a measurable $\mathrm{Li}$, which is in agreement with the expected fraction of field stars without Li (see next section).

The remaining 6 contaminating field stars show the Li line but cannot be identified in the sample of the 29 members shown in Fig. 6b.

In Fig. 8 we compare the Li abundances for the 29 NGC 3960 candidate members with the $\mathrm{Li}$ abundances of the Hyades. The two distributions are almost identical for stars warmer than about $6000 \mathrm{~K}$, while the Li abundances of NGC 3960 stars cooler than $6000 \mathrm{~K}$ are systematically smaller than those of Hyades members of similar temperature. This result on the one hand supports our initial assumption that NGC 3960 is older than the Hyades; on the other hand, it allows us to add a critical datapoint (see Sect. 1) on the empirical study of the evolution of $\mathrm{Li}$ abundance with age.

Figure 9, adapted from Sestito \& Randich (2005), shows the average $\mathrm{Li}$ abundances as a function of age, in three different effective temperature ranges, as computed by Sestito \& Randich (2005) for open clusters of different ages; the average values in the same temperature ranges derived here for NGC 3960 using the $29 R V$ members with $\mathrm{Li}$ are indicated in the figure and are given in Table 2. For comparison, the position of the Sun is also indicated.

For stars cooler than $5700 \mathrm{~K}$ (Fig. 9c), the average Li abundance in NGC 3960 is computed from only two stars and the associated error is the semi-difference of the two Li abundances.

\footnotetext{
3 The sample of the 10 stars includes the binary with a $R V$ consistent with that of the cluster, for which the $E W$ has not been measured.
} 
Table 2. Average of $\log n(\mathrm{Li})$ for NGC 3960 in three different ranges of effective temperatures.

\begin{tabular}{cccc}
\hline \hline$\Delta T_{\text {eff }}$ & $\langle\log n(\mathrm{Li})\rangle$ & error & error type \\
\hline$[6050-6350] \mathrm{K}$ & 2.72 & 0.25 & $\sigma$ \\
{$[5750-6050] \mathrm{K}$} & 2.19 & 0.48 & $\sigma$ \\
{$[5500-5700] \mathrm{K}$} & 1.88 & 0.35 & maximum error \\
\hline
\end{tabular}

As discussed in Sestito \& Randich (2005), the upper panel shows that after a small amount of Li depletion occurring during the pre-main sequence (PMS) phase, no Li destruction is present up to about $250 \mathrm{Myr}$, while it restarts. The average abundances for NGC 3960 confirm the trend of slow (but present) depletion at ages older than the Hyades.

Figure $9 \mathrm{~b}$ and $\mathrm{c}$ indicate that for cooler stars, after the phase of PMS Li destruction, Li depletion restarts significantly after about 200 Myr. Importantly, our analysis allows us to put tighter constraints on the age at which Li depletion is no longer present. Specifically, the average Li abundances derived for NGC 3960 shows that the Li plateau might start at $\sim 1$ Gyr rather than 2 Gyr: the average abundance of NGC 3960 is indeed closer to that of the 2 Gyr clusters, rather than to the Hyades, although, due to the large $\sigma$, it is not completely inconsistent with the latter. Also, average Li abundances in NGC 3960 could be lowered by the inclusion of a few non-members with $R V$ s consistent with that of NGC 3960 but, which might have lower Li abundances. For example, the average $\mathrm{Li}$ abundance of stars in Fig. $9 \mathrm{~b}$ has been computed, including in the sample the star with $\log n(\mathrm{Li}) \simeq 1.15$ and $T_{\text {eff }} \simeq 5800 \mathrm{~K}$; its $\mathrm{Li}$ abundance significantly deviates from the mean Li pattern, suggesting that this object might come from the sample of contaminating field stars. By excluding this object from the sample we would get a smaller $\sigma(0.31)$ and a slightly higher average (2.32), which is however consistent with that of older clusters.

We stress that our analysis has been carried out consistently with that of Sestito \& Randich (2005) and thus the comparison of the average abundances should not be affected by systematic errors.

As discussed by Sestito \& Randich (2005), none of the models/mechanisms proposed to explain the occurrence of MS Li depletion is also able to reproduce the plateau, since all of them predict that Li depletion should continue at old ages. To our knowledge, no new models predicting the existence of the plateau have been presented. Our study, not only reinforces the empirical evidence for the plateau and thus the need for such models, but also provides an additional constraint to be taken into account.

\subsubsection{Field stars}

Figure $6 \mathrm{c}$ shows the $\mathrm{Li}$ abundance distribution for the 26 objects that are contaminating field stars according to their $R V$, but do show the Li line. Their distribution is not remarkably different from that of NGC 3960 members, although it is characterized by a much larger dispersion. As discussed in Prisinzano et al. (2004), the differential reddening correction was computed as the distance (along the reddening vector) of the position in the CMD of each star from the assumed MS at the cluster distance. Therefore, such correction does not take into account the distance spread from the Sun for field stars and this explains the large spread in the Li abundance.

Nevertheless, several stars with high abundance $(\log n(\mathrm{Li})>$ 2) are present. More specifically, we find that the field stars hotter

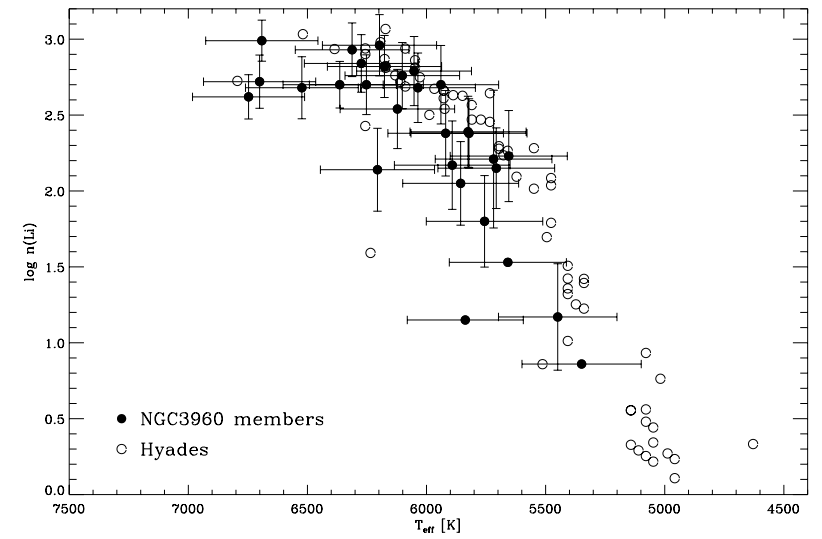

Fig. 8. Li abundances of the 29 NGC 3960 members (filled circles) as in Fig. 6 (panel b)), compared with the Hyades values (empty circles).

than about $6400 \mathrm{~K}$ (early F-type) show a Li abundance of about 2.7 , consistent with that found for stars of similar temperatures for NGC 3960; stars cooler than $6400 \mathrm{~K}$ and hotter than $5700 \mathrm{~K}$ (late F-type and early G-type) show a large spread in the Li abundance with values similar or smaller than those found for the same temperature range for NGC 3960; finally, stars with temperatures smaller than about $5500 \mathrm{~K}$ have $\mathrm{Li}$ abundances somewhat larger than those found for NGC 3960. Note, however, that effective temperatures (and thus Li abundances) for these stars might have been overestimated, since we have assumed the same reddening as for NGC 3960 members.

We exclude that the stars in Fig. 6c are binary cluster members (SB1) with discrepant $R V \mathrm{~s}$ due to the orbital component, since for these objects, the distribution of the difference between the maximum and the minimum values of the $R V$ measured from our spectra (acquired within about one and a half months, see Table 1) is very similar to that obtained for the sample of $R V$ members. Therefore, we conclude that they are, most likely, a sample of field stars with Li.

If we consider that we have 74 non-members for $R V$ (i.e. 113-39), including the binary, plus 16 contaminating field stars stars with $R V \mathrm{~s}$ consistent with that of NGC 3960, we have a total of 90 field stars; those with the Li line are 26 within the sample of $R V$ non members, plus the remaining 6 contaminating field stars in the sample of $R V$ members. Therefore, the fraction of field stars with the Li line is $(26+6) / 90$, i.e., about one third of the sample. This fraction is slightly lower than that found by Pasquini et al. (1994), who found that about one half of the $\mathrm{G}$ dwarfs analyzed in their work had high Li content $(2.0 \lesssim \log n(\mathrm{Li}) \lesssim 3.0)$ and apparently old age. Also note that all solar-type stars in the very old cluster NGC 188 ( $\sim 6$ Gyr) with available Li measurements have abundances above 2.0 (Randich et al. 2003), i.e., a relatively high $\mathrm{Li}$ is not inconsistent with a very old age.

In summary, the presence of several Li-rich F and G-type field stars in our sample is not surprising. The large spread in their Li abundance is both consistent with a mixed population of stars older or similar to NGC 3960, and also possibly/in part due to the fact that, in addition to stellar age, another unknown parameter can regulate MS Li depletion (e.g. Pasquini et al. 1994; Pinsonneault 1997; Pasquini et al. 1997; Charbonnel \& Talon 2005; Randich et al. 2006, and references therein). 

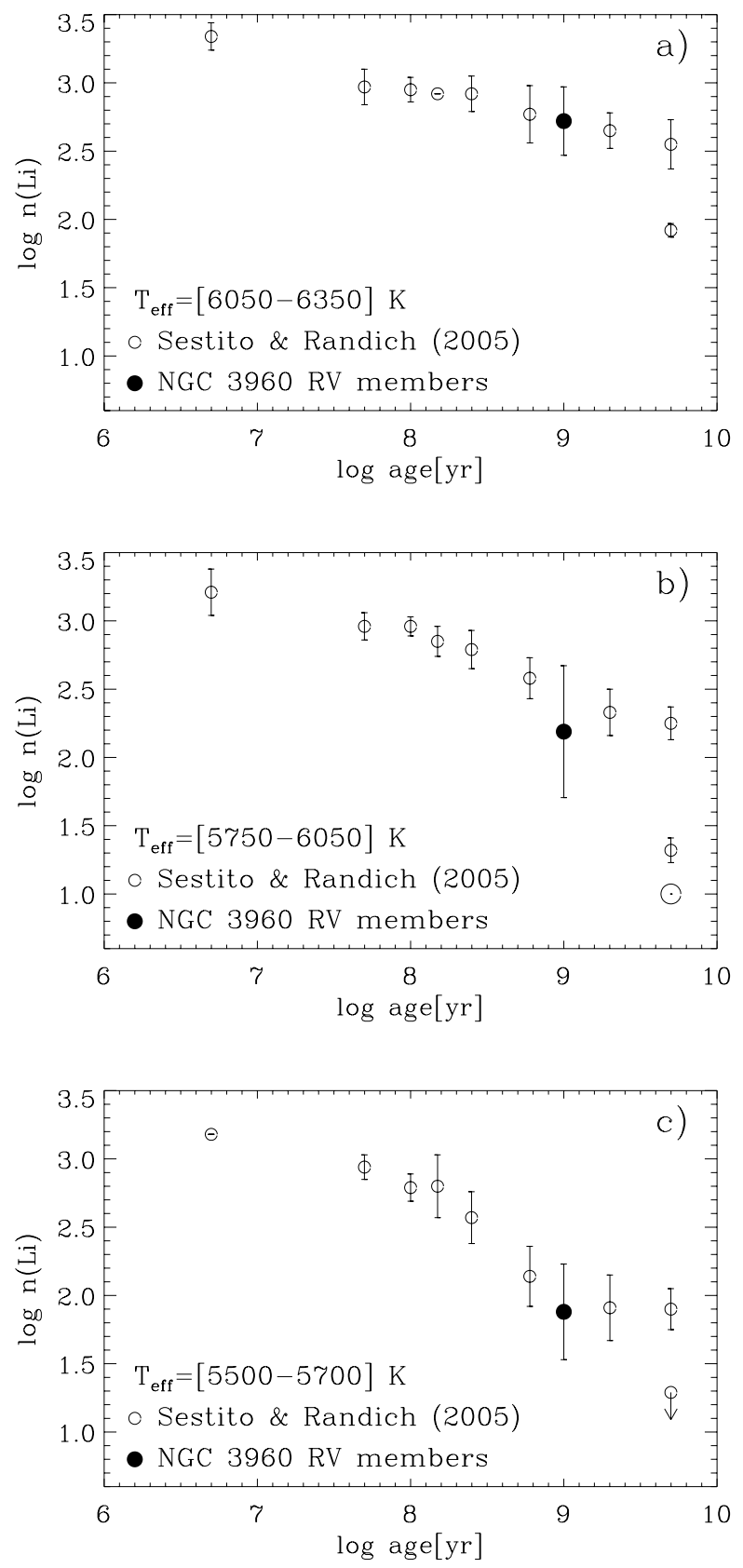

Fig. 9. Plots adapted from Fig. 7 of Sestito \& Randich (2005) showing the average $\log n(\mathrm{Li})$ as a function of cluster age, in three different temperature ranges. Open circles are the data from Sestito \& Randich (2005), while filled circles are the values computed for NGC 3960.

\subsection{Membership and contamination}

Table 3 gives the total number of stars defined as cluster members or non-members based on the $R V$ and the presence or not of the Li line in their spectrum. As already discussed in the previous section, we find a total of 29 objects that satisfy both membership criteria. This sample contains 6 of the 16 contaminating stars with $R V$ s consistent with that of NGC 3960, estimated by the $R V$ distribution of the field stars (see Sect. 3.1). The remaining 10 contaminating stars can be individually distinguished since they do not show the Li line. If we add the 74 stars with $R V$ s not consistent with the cluster membership, we have a total of 84 individually known non-members over a total of 113 observed stars.
Table 3. Number of stars with $R V$ consistent or not (within $3 \sigma$ ) with the cluster membership (indicated by $Y$ or $N$ ) and with or without $\mathrm{Li}$ (indicated by $Y$ or $N$ ). The total number for each criterion is also given. The two binaries are specifically indicated in the given samples.

\begin{tabular}{lc|ccc}
\hline \hline $\mathrm{Li}$ & $R V$ & $Y$ & $N$ & Tot. \\
\hline$Y$ & & 29 & 26 & 55 \\
$N$ & & $10(9+1)$ & $48(47+1)$ & $58(56+2)$ \\
Tot. & & $39(38+1)$ & $74(73+1)$ & $113(111+2)$ \\
\hline
\end{tabular}

We considered these 84 objects to estimate the fraction of contaminating field stars in our sample as a function of the $V$ magnitude. The results are shown by the solid line histogram plotted in Fig. 10; the number of contaminating stars and the total number of objects within the four magnitude ranges are also indicated; error bars were computed from the binomial distribution. The resulting fractions are compared with the analogous values derived in Prisinzano et al. (2004), where the contamination was statistically derived using a field region outside the cluster region; in this case, errors were computed from the propagation of the poisson errors on the number of field stars and of the total stars. The comparison shows that, within the errors, the fractions of contaminating stars derived with the two methods are compatible.

We note, however, that, among the 84 contaminating stars, the sample of 74 non-members for the $R V$ could include a small fraction of binaries of NGC 3960, therefore these numbers could be slightly overestimated. On the other hand, we did not include in the field star sample the 6 contaminating stars with $R V$ s consistent with that of the cluster and with the Li line. Nevertheless, since these effects should be within the error bars, we conclude that the fraction of contaminating field stars within the spectroscopic sample studied in this work is comparable with that estimated in Prisinzano et al. (2004) with photometric data. We note that the comparison is consistent since the stars observed in this spectroscopic work have been randomly selected from the list of photometric candidate members given in Prisinzano et al. (2004). This result allows us to confirm the mass function derived in Prisinzano et al. (2004), which strongly depends on the correction for the field star contamination.

\section{Summary and conclusions}

We used VLT/FLAMES Giraffe spectra to determine the $R V \mathrm{~s}$ and thus the membership of a sample of 113 photometrically selected candidate cluster members. We find that the average cluster $R V$ is $-20.0 \pm 0.7 \mathrm{~km} \mathrm{~s}^{-1}$ with a standard deviation $\sigma=$ $2.3 \pm 0.6 \mathrm{~km} \mathrm{~s}^{-1}$. As expected from the high fraction of contaminating stars, only 39 objects have $R V$ s consistent with that of the cluster. Among these, 16 are expected to be contaminating field stars and 10 of these have been identified since they do not show the Li line. We find that the spread in the CMD for the sample of cluster members is not reduced since the adopted reddening correction is statistically reliable. Individual spectral types of such objects are necessary to accurately derive their temperatures and positions in the CMD.

From the analysis of the Li line we derived $\mathrm{Li}$ abundances for cluster and field stars. We find that by using photometry corrected for differential reddening, $\mathrm{Li}$ abundance distribution of the cluster members shows a spread smaller than that found using uncorrected photometry. 


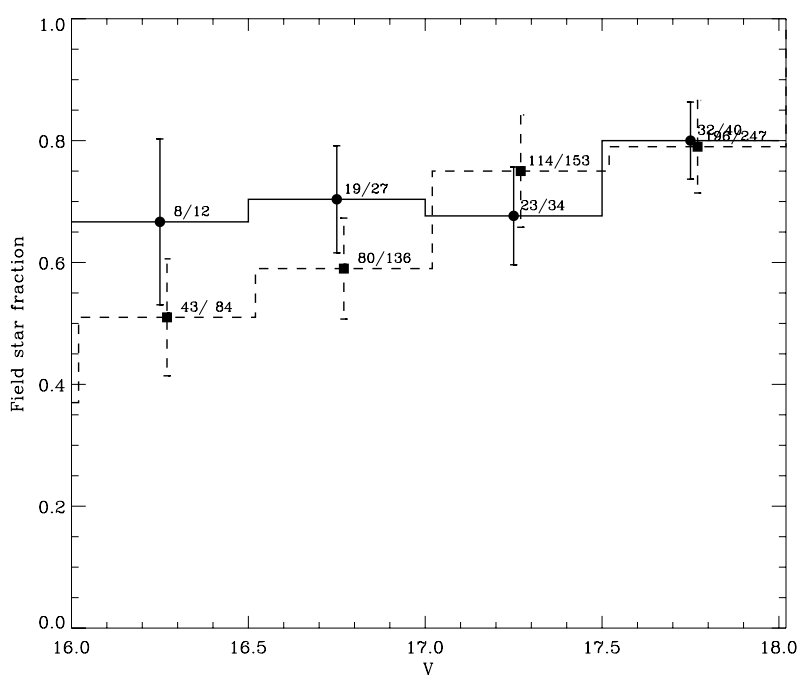

Fig. 10. Fraction of contaminating field stars estimated in this work from individual membership derived by the $R V$ and the Li line (solid line histogram) and in Prisinzano et al. (2004) from a statistical analysis of a field region around NGC 3960 (dashed line histogram).

The average Li abundances computed for stars in three different temperature ranges confirms the trend, already found in Sestito \& Randich (2005), of slow (but present) depletion at ages older than the Hyades for stars with temperatures larger than about $6000 \mathrm{~K}$. For cooler stars, the inclusion of the average $\mathrm{Li}$ abundance of NGC 3960 in the distribution of Li abundance as a function of the age, allows us to conclude that the age at which $\mathrm{Li}$ depletion is no longer present is very likely closer to $1 \mathrm{Gyr}$ rather than 2 Gyr, as inferred by Sestito \& Randich (2005), on the base of the incomplete sample of open clusters with ages older than 2 Gyr and younger than the Hyades.

The fraction of field stars with an evident $\mathrm{Li}$ line is about one third, while the fraction of field stars, as a function of the magnitude, is consistent with that derived statistically from photometry. This allows us to confirm the conclusions about the mass function derived in Prisinzano et al. (2004), which strongly depends on the estimate of the field star contamination.
Acknowledgements. We wish to thank the referee R. D. Jeffries for his useful suggestions which have improved the final version of this paper. We also thank the ESO Paranal staff for performing the service mode observations. This work has made extensive use of the services of WEBDA, ADS, CDS etc. The research presented here has been supported by an INAF grant on Stellar clusters as probes of star formation and early stellar evolution (PI: F. Palla). L.P. thanks Paolo Spanò for his contribution to the $R V$ analysis and Giusi Micela for useful discussions on this work.

\section{References}

Blecha, A., \& Simond, G. 2004, Technical report (GIRAFFE BLDR Software Reference Manual Version 1.12, Observatoire de Geneve)

Bonatto, C., \& Bica, E. 2006, A\&A, 455, 931

Bragaglia, A., Tosi, M., Carretta, E., et al. 2006, MNRAS, 366, 1493

Carlsson, M., Rutten, R. J., Bruls, J. H. M. J., \& Shchukina, N. G. 1994, A\&A, 288,860

Charbonnel, C., \& Talon, S. 2005, Science, 309, 2189

Friel, E. D., \& Janes, K. A. 1993, A\&A, 267, 75

Jeffries, R. D., \& Oliveira, J. M. 2005, MNRAS, 358, 13

Pallavicini, R., Spanò, P., Prisinzano, L., et al. 2006, Multi-Object Spectroscopy of Open Clusters with FLAMES: Preliminary GTO Results, Chemical Abundances and Mixing in Stars in the Milky Way and its Satellites, ESO Astrophysics Symposia (Springer-Verlag), 181

Pasquini, L., Liu, Q., \& Pallavicini, R. 1994, A\&A, 287, 191

Pasquini, L., Randich, S., \& Pallavicini, R. 1997, A\&A, 325, 535

Pinsonneault, M. 1997, ARA\&A, 35, 557

Prisinzano, L., Micela, G., Sciortino, S., \& Favata, F. 2004, A\&A, 417, 945

Prisinzano, L., Damiani, F., Micela, G., \& Pillitteri, I. 2007, A\&A, 462, 123

Randich, S., Sestito, P., \& Pallavicini, R. 2003, A\&A, 399, 133

Randich, S., Bragaglia, A., Pastori, L., et al. 2005, The Messenger, 121, 18

Randich, S., Sestito, P., Primas, F., et al. 2006, A\&A, 450, 557

Sestito, P., \& Randich, S. 2005, A\&A, 442, 615

Sestito, P., Bragaglia, A., Randich, S., et al. 2006, A\&A, 458, 121

Soderblom, D. R., Oey, M. S., Johnson, D. R. H., \& Stone, R. P. S. 1990, AJ, 99, 595

Soderblom, D. R., Jones, B. F., Balachandran, S., et al. 1993a, AJ, 106, 1059

Soderblom, D. R., Stauffer, J. R., Hudon, J. D., \& Jones, B. F. 1993b, ApJS, 85, 315

Soderblom, D. R., Jones, B. F., Stauffer, J. R., \& Chaboyer, B. 1995, AJ, 110, 729

Sozzetti, A., Torres, G., Charbonneau, D., et al. 2007, ApJ, 664, 1190

Thorburn, J. A., Hobbs, L. M., Deliyannis, C. P., \& Pinsonneault, M. H. 1993, ApJ, 415, 150

Tonry, J., \& Davis, M. 1979, AJ, 84, 1511 
L. Prisinzano and S. Randich: Lithium abundances in the old open cluster NGC 3960, Online Material p 1

\section{Online Material}


L. Prisinzano and S. Randich: Lithium abundances in the old open cluster NGC 3960, Online Material p 2

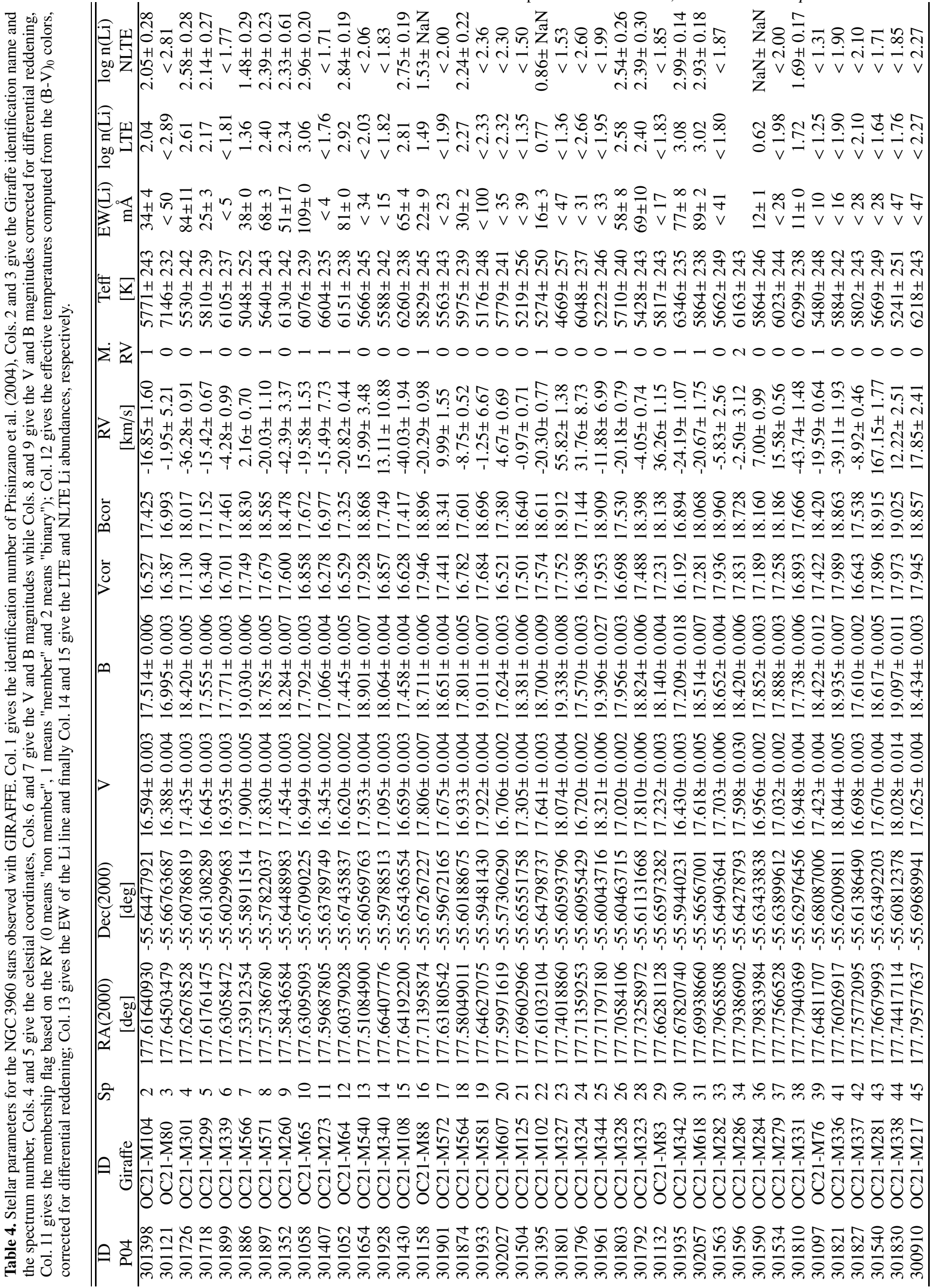


L. Prisinzano and S. Randich: Lithium abundances in the old open cluster NGC 3960, Online Material p 3

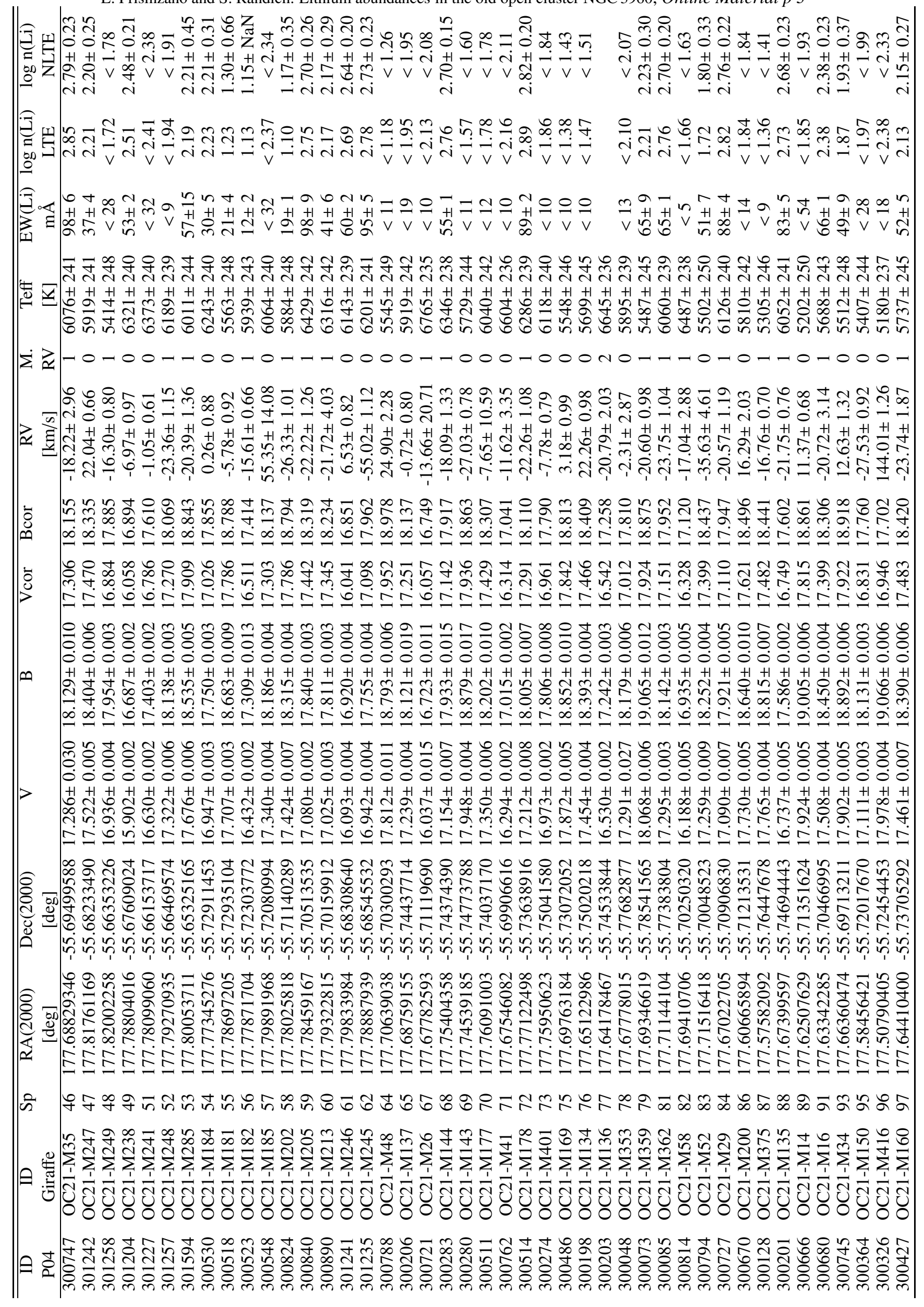


L. Prisinzano and S. Randich: Lithium abundances in the old open cluster NGC 3960, Online Material p 4

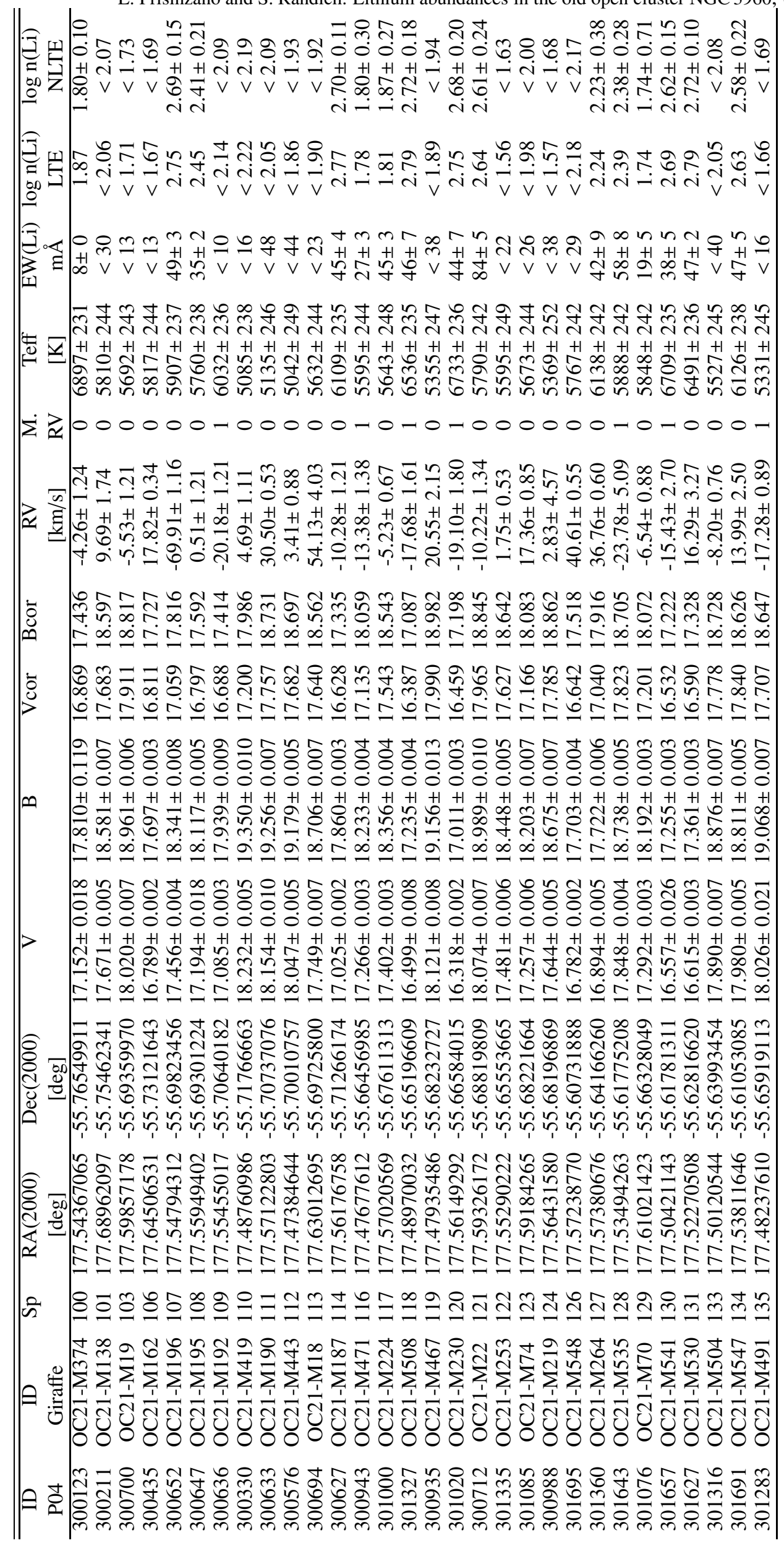

